\title{
Challenges with colorectal cancer staging: results of an international study
}

\author{
Dipti M. Karamchandani ${ }^{1} \cdot$ Runjan Chetty $\mathbb{D}^{2} \cdot$ Tonya S. King $^{3} \cdot$ Xiuli Liu $^{4} \cdot$ Maria Westerhoff $^{5} \cdot$ Zhaohai Yang $^{1,8}$. \\ Rhonda K. Yantiss ${ }^{6}$. David K. Driman ${ }^{7}$
}

Received: 22 April 2019 / Revised: 22 July 2019 / Accepted: 23 July 2019 / Published online: 5 August 2019

(c) United States \& Canadian Academy of Pathology 2019

\begin{abstract}
Challenges exist with standardized colorectal cancer reporting despite adoption of the American Joint Committee on CancerStaging Manual 8th edition. We performed this study to gauge current practice patterns among a diverse group of surgical pathologists. A web-based questionnaire depicting problematic issues and images related to colorectal carcinoma staging was circulated among 118 surgical pathologists and their responses were correlated with their geographic location (North America vs. Europe vs. others), nature of practice (academic vs. community), the sign-out model (gastrointestinal subspecialty vs. general surgical pathology), and years of professional experience. We found that a substantial number of practicing pathologists ignore recommended-staging criteria in specific settings, particularly with respect to assessment of advanced T stage. Tumors that communicated with the serosa through inflammatory foci were staged as pT3 (49\%) or pT4a $(51 \%)$ by nearly equal numbers of pathologists regardless of level of experience, the sign-out model, or geographic location. Only 65\% assigned $\mathrm{T}$ stage and margin status based on extent of viable tumor in the neoadjuvant setting. One-third of pathologists, particularly those in Europe $(p=0.015)$, classified acellular mucin deposits as N1 disease when detected in treatment-naive cases. Nearly $50 \%$ of pathologists classified isolated tumor cells (i.e., deposits $<0.2 \mathrm{~mm}$ ) in lymph nodes as metastatic disease (i.e., pN1, $p=0.02$ ). Our results suggest that pathologists ignore recommendations that are based on insufficient data and apply individualized criteria when faced with situations that are not addressed in the American Joint Committee on Cancer Staging Manual 8th edition. These variations in practice limit the ability to compare outcome data across different institutions and draw attention to areas that require further study.
\end{abstract}

Portions of this study were presented as platform and poster at the United States and Canadian Academy of Pathologists annual 2019 meeting held at National Harbor, Maryland.

$\triangle$ Dipti M. Karamchandani

dkaramchandani@pennstatehealth.psu.edu

1 Department of Pathology, Penn State Health Milton S. Hershey Medical Center/Penn State College of Medicine, Hershey, PA, USA

2 Department of Pathology, Laboratory Medicine Program, University Health Network, Toronto, ON, Canada

3 Department of Public Health Sciences, Penn State College of Medicine, Hershey, PA, USA

4 Department of Pathology, Immunology and Laboratory Medicine, University of Florida, Gainesville, FL, USA

\section{Introduction}

Colorectal carcinoma is the second most common cause of cancer-related death in men and women, and the most common cause of cancer-related death among nonsmokers. There will be $\sim 51,020$ deaths in the United States attributable to colorectal carcinoma in 2019 , as well as $\sim 101,420$ newly

5 Department of Pathology, University of Michigan, Ann Arbor, MI, USA

6 Department of Pathology and Laboratory Medicine, Weill Cornell Medical College, New York, NY, USA

7 Department of Pathology and Laboratory Medicine, Western University and London Health Sciences Centre, London, ON, Canada

8 Present address: Department of Pathology \& Laboratory Medicine, University of Pennsylvania Perelman School of Medicine, Philadelphia, PA, USA 
diagnosed colon cancers and 44,180 new rectal cancers [1]. Treatment options for these patients are largely dictated by tumor stage, as determined by the tumor, node, metastasisstaging system. This system was developed by the American Joint Committee on Cancer and the Union for International Cancer Control to provide uniform criteria for cancer staging and, unlike previous staging systems, is updated as new prognostic and therapeutic information accumulates [2, 3]. However, the system is not designed to address every possible cancer-staging scenario, especially when compelling data are not available. As a result, staging recommendations largely reflect the authors' opinions in some situations and completely fail to address others, leading some practicing pathologists to challenge poorly supported staging criteria or offer their own interpretations for handling difficult issues [4-9]. The purpose of this study was to assess how pathologists apply cancer-staging criteria when faced with problematic issues that are not well delineated or supported by data in the American Joint Committee on Cancer 8th edition Staging Manual. We also assessed whether reporting practices were influenced by regional effects, different practice settings, or years of experience.

\section{Materials and methods}

A web-based questionnaire was circulated among an international group of surgical pathologists over a span of one month (August 1-31, 2018). The questionnaire was circulated to selected professional committees in an attempt to target like-minded pathologists (such as United States and Canadian Academy of Pathology Gastrointestinal Pathology Society website), and via social media (such as pathology Facebook group). The authors of the study also forwarded the survey via email to colleagues practicing in the same and/or different regions of the world who they believed would be interested in taking this survey and/or encounter such cases in their clinical practice. Participation was voluntary. The survey results were anonymous, and no identifiers were associated with this survey. The survey completion rate is unknown. The respondents were asked to classify their own practices as academic gastrointestinal pathology subspecialists, academic generalists, private practitioners, or trainees. They also reported the countries in which they resided and number of years in clinical practice.

The survey contained case scenarios and questions to gauge practices with respect to staging colorectal cancers from treatment-naive and neoadjuvantly treated patients. The case scenarios and questions in the survey pertaining to staging treatment-naive colorectal carcinomas are presented in Table 1. Specific issues included the distinction between pathologic stages T3 and T4a, classification of isolated tumor cells (i.e., deposits $<0.2 \mathrm{~mm}$ ) in lymph nodes, and assessment

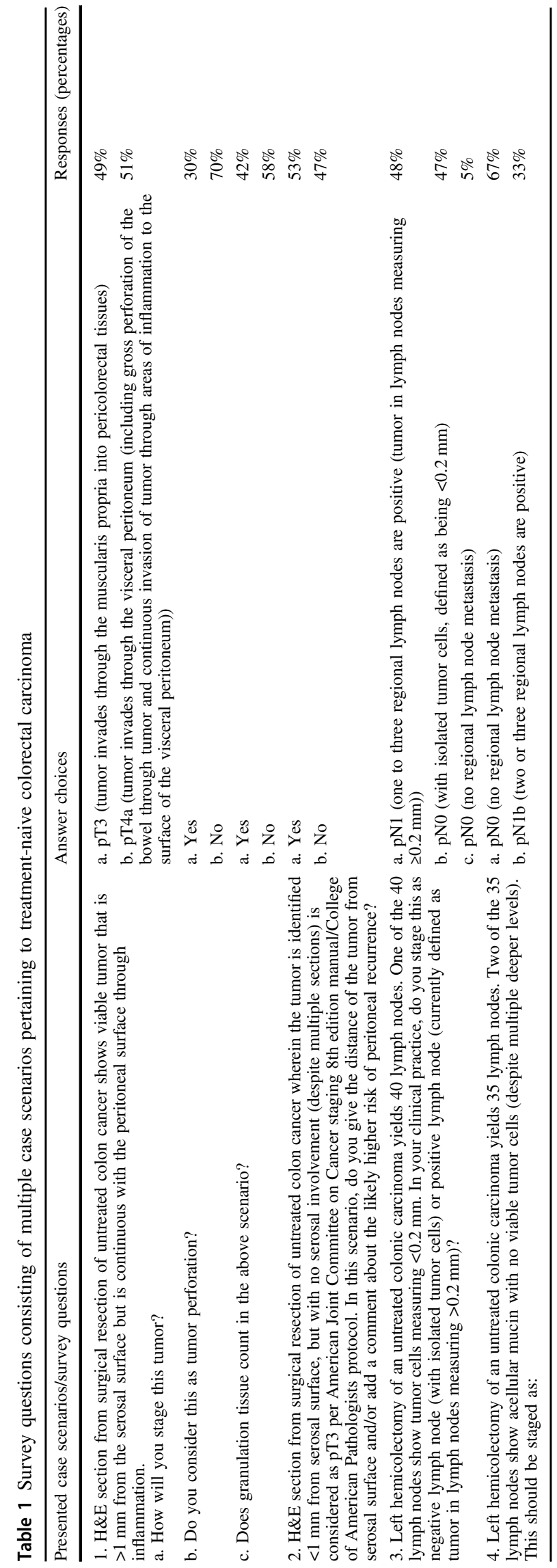


of acellular mucin deposits in lymph nodes accompanying treatment-naive tumors. The case scenarios and questions in the survey targeted to staging of neoadjuvantly treated rectal carcinomas are presented in Table 2. Figures were also provided with selected case scenarios (i.e., for Q1-Q6 in Table 2; only selected pictures included in this paper). Questions pertaining to tumor stage, radial margin status, and lymph node metastases when acellular mucin pools were present in tumors from neoadjuvantly treated patients were presented. We also queried practices with respect to tumor budding, intramucosal carcinoma, and histologic classification of mucinous histology in neoadjuvantly treated cancers. Responses were tabulated and analyzed according to practice setting, geography, and years of practice.

\section{Statistical analysis}

Associations between the categorical demographic variables and survey question responses were evaluated using Chisquare or Fisher's exact tests and reported with corresponding frequencies and percentages for comparison. The cluster analysis was performed using Ward's minimum-variance hierarchical-clustering method employing an agglomerative approach. Samples were merged into larger clusters at each generation of clusters to minimize the within-cluster sum of squares or to maximize the between-cluster sum of squares. Chi-square tests were used to compare the distribution of demographic variables among the clusters. The analyses were performed using SAS v9.4, SAS Institute Inc., Cary, NC, and a $p$-value $<0.05$ was considered significant.

\section{Results}

The survey was completed by 118 pathologists over a span of 1 month (August 1-31, 2018). Fifty-eight (49\%) respondents practiced in an academic setting with a subspecialized signout model, 32 (27\%) were generalists practicing in an academic setting, and the remainder were private practitioners $(n=22,19 \%)$ or trainees $(n=6,5 \%)$. Sixty-six $(56 \%)$ respondents were from North America, including 60 (51\%) from the United States. Twenty-two (19\%) were from Europe and $21(18 \%)$ were from Asia; the rest were located in Australia (6\%) and Africa (1\%). Forty-eight (43\%) respondents had more than 10 years of clinical experience and $25 \%$ had 5-10 years of clinical experience.

\section{Treatment-naive colorectal carcinoma (Table 1)}

\section{Staging challenges T3 vs. T4a (Q1a-c, Q2)}

Although the American Joint Committee on Cancer 8th edition Cancer Staging Manual classifies tumors that communicate with the serosa via inflammation as pT4a, 49\% respondents considered this finding to represent a pT3 lesion (Fig. 1). There were no differences with respect to practice model, region, or years of experience; $50 \%$ of academic generalists and subspecialists and $55 \%$ of private practitioners classified this finding as pT4a, as did $53 \%$ of pathologists in the United States, $48 \%$ of pathologists outside the United States, and $44 \%$ of pathologists with more than 10 years of experience. Of note, $30 \%$ of respondents considered tumor cells communicating with the serosa through an inflammatory focus to represent tumor perforation. Most (44\%) of these individuals were academic generalists, $24 \%$ were academic gastrointestinal pathology subspecialists, and $23 \%$ were private practitioners with similar amounts of clinical experience (0-5 years: 25\%, 5-10 years: $32 \%$, >10 years: $31 \%$ ). There was no relationship between response and practice setting $(p=0.11)$, years of experience $(p=0.8)$, or practice region $(p=0.12)$. A substantial number $(42 \%)$ of pathologists considered granulation tissue to be equivalent to inflammation when distinguishing pT3 from pT4: $38 \%$ of academic generalists, $42 \%$ of academic subspecialists, and $50 \%$ of private practitioners classified a tumor that communicated with the serosa through an area of granulation tissue as a pT4 lesion (Fig. 1). There was no relationship between opinion on this matter and clinical experience (0-5 years of experience: $44 \%$, 5-10 years: $41 \%,>10$ years: $42 \%$ ) or practice region ( $41 \%$ US pathologists vs.s $43 \%$ of non-US pathologists). Most (53\%) pathologists also provided clinical colleagues with information regarding tumors $<1 \mathrm{~mm}$ from the serosa, routinely noting a higher risk of peritoneal recurrence and the distance between the tumor and serosa. These respondents represented all practice settings $(59 \%$ of academic generalists, $47 \%$ of subspecialists practicing in academic setting, and $55 \%$ of private practitioners), and had variable clinical experience (61\% with $0-5$ years, $39 \%$ with $5-10$ years, and 52\% with $>10$ years).

Diagnosis of isolated tumor cells in nodal metastases (Q3)

Almost half (48\%) of all respondents disregarded recommendations to classify isolated tumor cells $(<0.2 \mathrm{~mm}$ deposits) as pN0 and considered this finding to represent pN1 disease (Fig. 2a). There were no significant differences with respect to years of experience $(p=0.2)$ or country of practice $(p=0.6)$, although academic generalists $(69 \%)$ diagnosed this scenario significantly more as $\mathrm{pN} 1(p=0.02)$ when compared with subspecialists practicing in academic setting (40\%), and private practitioners $(32 \%)$. 


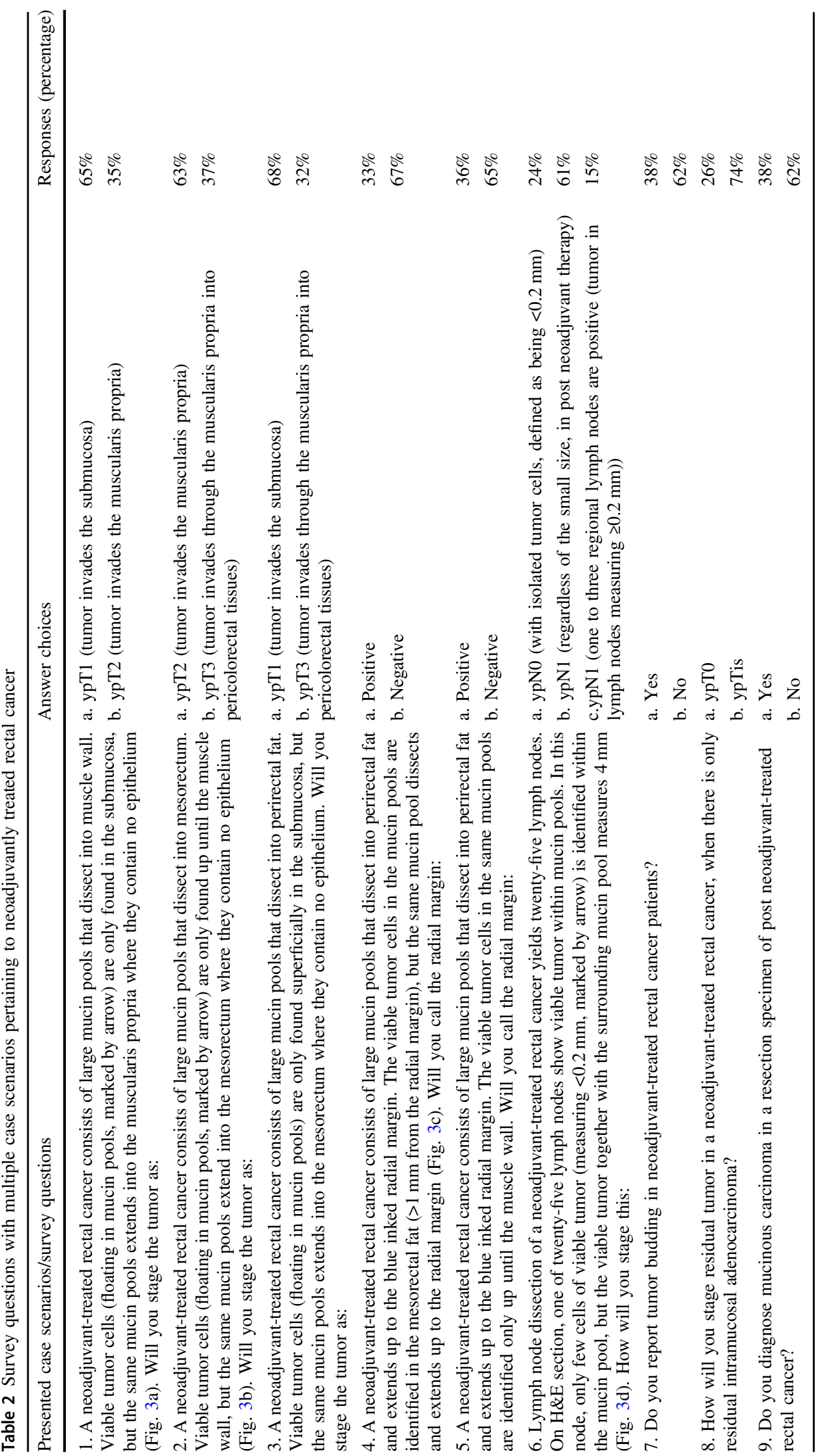




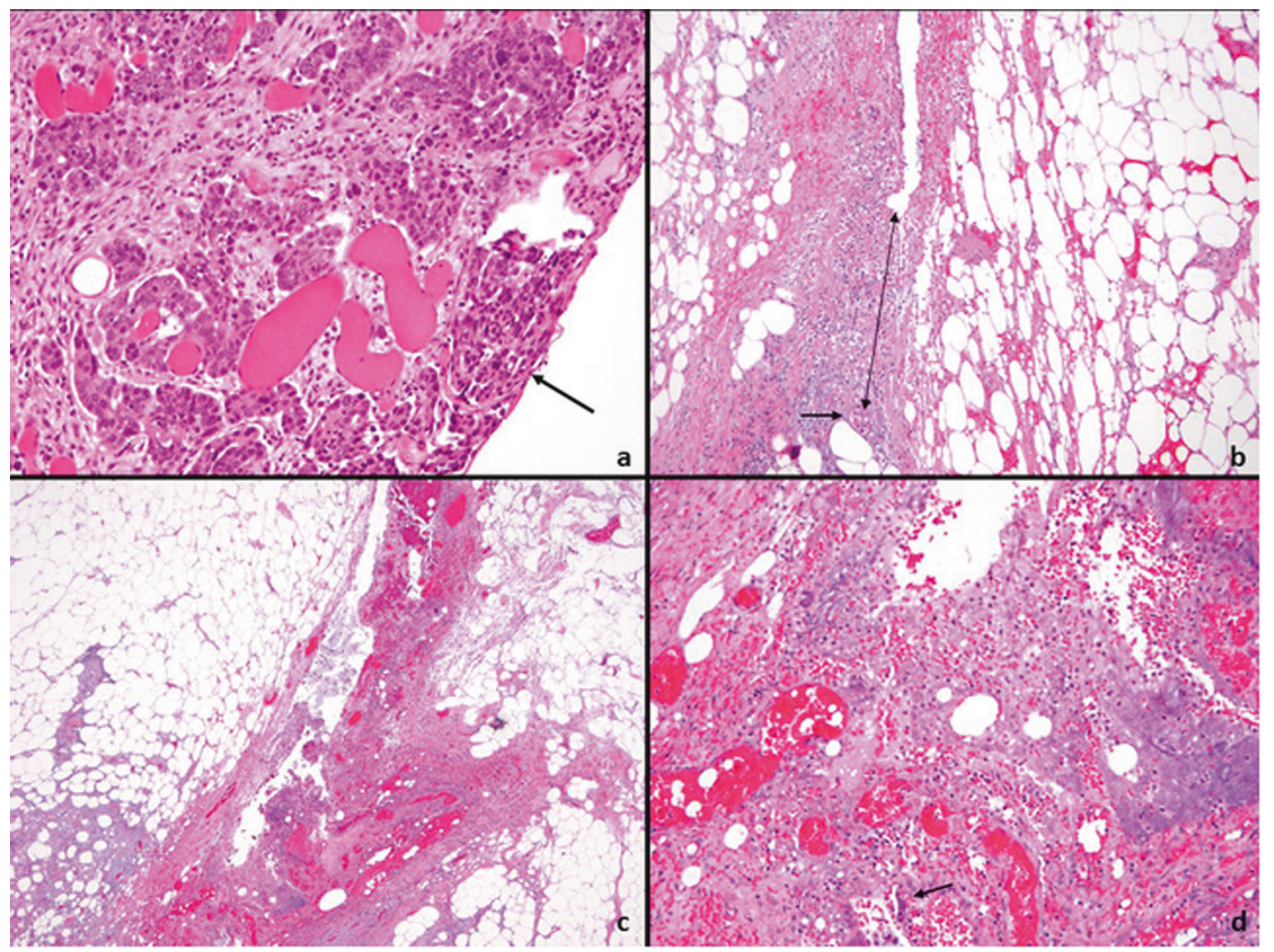

Fig. 1 Staging dilemma between pT4a vs. pT3 in treatment-naive colorectal carcinoma. a Unequivocal T4a where tumor cells are identified at the serosal surface (marked by arrow). b-d Tumor cells (marked by arrow) separated from the serosal surface by only granulation tissue in photomicrograph (b). Tumor cells, marked by arrow in photomicrograph (d), present at a distance from serosal surface with

\section{Assessment of acellular mucin deposits in lymph nodes (Q4)}

One-third of surveyed pathologists considered acellular mucin deposits in lymph nodes of untreated patients (Fig. 2b) to represent $\mathrm{pN} 1$ disease, regardless of practice setting or years of experience. However, more European pathologists $(59 \%)$ considered this finding to represent pN1 disease than did non-European pathologists (30\%; $p=0.015$ ).

\section{Neoadjuvantly treated rectal carcinoma (Table 2)}

\section{Depth of invasion (Q1-3)}

Sixty-five percent of respondents assigned a T-stage based on the deepest extent of viable tumor cells, whereas $35 \%$ used the deepest extent of mucin to determine $\mathrm{T}$ stage (Fig. 3a, b). There was a significant relationship ( $p=$ 0.04 ) between stage assignment and different practice types: $71 \%$ of academic subspecialists and $64 \%$ of private intervening abscess and granulation tissue that communicates with the serosal surface in photomicrographs (c) and (d). Fifty-one percent respondents staged these as pT4a, and 49\% assigned pT3. (a-d H\&E $\times 200,100,40$, and 200 , respectively; d represents a higher power photomicrograph of c)

practitioners assigned tumor stage based on extent of viable tumor only, compared with only $44 \%$ of academic generalists who assigned T-stage based on depth of mucin extension. There was no significant relationship between staging practices and years of clinical experience $(0-5$ years: $69 \%, 5-10$ years: $71 \%,>10$ years: $56 \% ; p=0.30$ ). However, pathologists in the United States were more likely to assign stage based on depth of viable tumor focus than those from other countries $(72 \%$ vs. $54 \%, p=0.04)$.

\section{Radial margin assessment $(\mathrm{Q} 4,5)$}

Most (65\%) of respondents considered acellular mucin present at the radial margin to represent a negative resection margin (Fig. 3c), although academic subspecialists (72\%) and private practitioners (73\%) were more likely to apply this criterion than academic generalists $(44 \%, p=0.02$ for both comparisons). Pathologists from the United States were also more likely to classify such cases as completely resected compared with individuals from other countries ( $78 \%$ vs. $50 \%, p=0.0013$ ). 


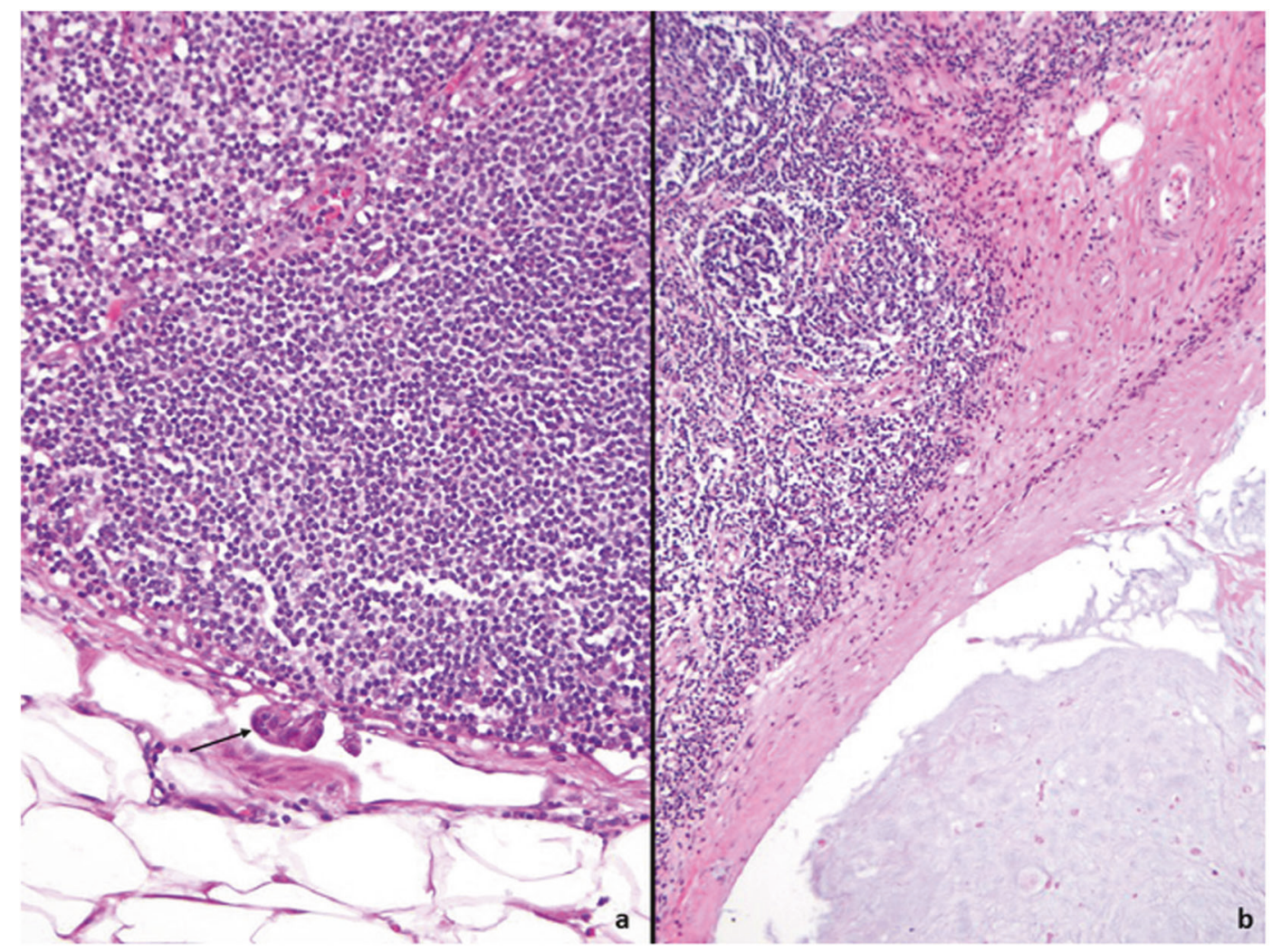

Fig. 2 Lymph node staging issues in treatment-naive colorectal carcinoma. (a) Isolated tumor cells spanning $<0.2 \mathrm{~mm}$ in aggregate (arrow) were staged as pNO(i+) by $47 \%$ of respondents, $48 \%$ assigned $\mathrm{pN} 1$, and $5 \%$ considered it to represent $\mathrm{pN} 0$ disease. b Acellular mucin

\section{Nodal metastases (Q6)}

Only 24\% of pathologists adhered to American Joint Committee on Cancer 8th edition guidelines regarding staging of small $(<0.2 \mathrm{~mm})$ tumor deposits in lymph nodes of neoadjuvant-treated rectal carcinomas. In fact, $76 \%$ of respondents ignored tumor deposit size in the neoadjuvant setting, considering any amount of tumor in the lymph node as ypN1 (Fig. 3d). There was no relationship between stage assessment and practice setting. Interestingly, pathologists with $<5$ years $(75 \%)$ and $>10$ years $(90 \%)$ of experience were more likely to classify such cases as ypN1 compared with those who had 5-10 years $(57 \%)$ of clinical experience $(p=0.02)$. Pathologists in the United States were more likely to consider such cases to be ypN0 (with isolated tumor cells) compared with pathologists elsewhere (32\% vs. $16 \%$, respectively, $p=0.03$ ).

\section{Other histologic features (Q7-9)}

Most (62\%) respondents across all groups did not diagnose mucinous carcinoma or report tumor budding in neoadjuvantly treated rectal carcinomas. The majority (74\%) in regional lymph node of treatment-naive tumor staged as pN0 by $67 \%$ of respondents and $33 \%$ classified this as pN1. (a-b H\&E $\times 200$ and 100 , respectively)

considered residual intramucosal carcinoma to represent ypTis disease rather than ypT0 in the neoadjuvant setting.

\section{Cluster analysis of responses for neoadjuvant therapy}

The pattern of survey results for neoadjuvant-treated rectal cancers could be clustered in two groups (Table 3). Academic subspecialists in the United States (cluster 2) were more likely to consider only the deepest extent of viable tumor cells when assigning $\mathrm{T}$ stage and classify the radial margin as negative if only acellular mucin was present. This group was less likely to report tumor budding and mucinous carcinoma in the neoadjuvant setting and more likely to classify isolated tumor cells as ypN0 (with isolated tumor cells) disease in the neoadjuvant setting.

\section{Discussion}

Tumor stage, as defined by tumor, node, metastasis-staging system, is the single most important factor-affecting patient survival and informs the use of adjuvant therapy and 


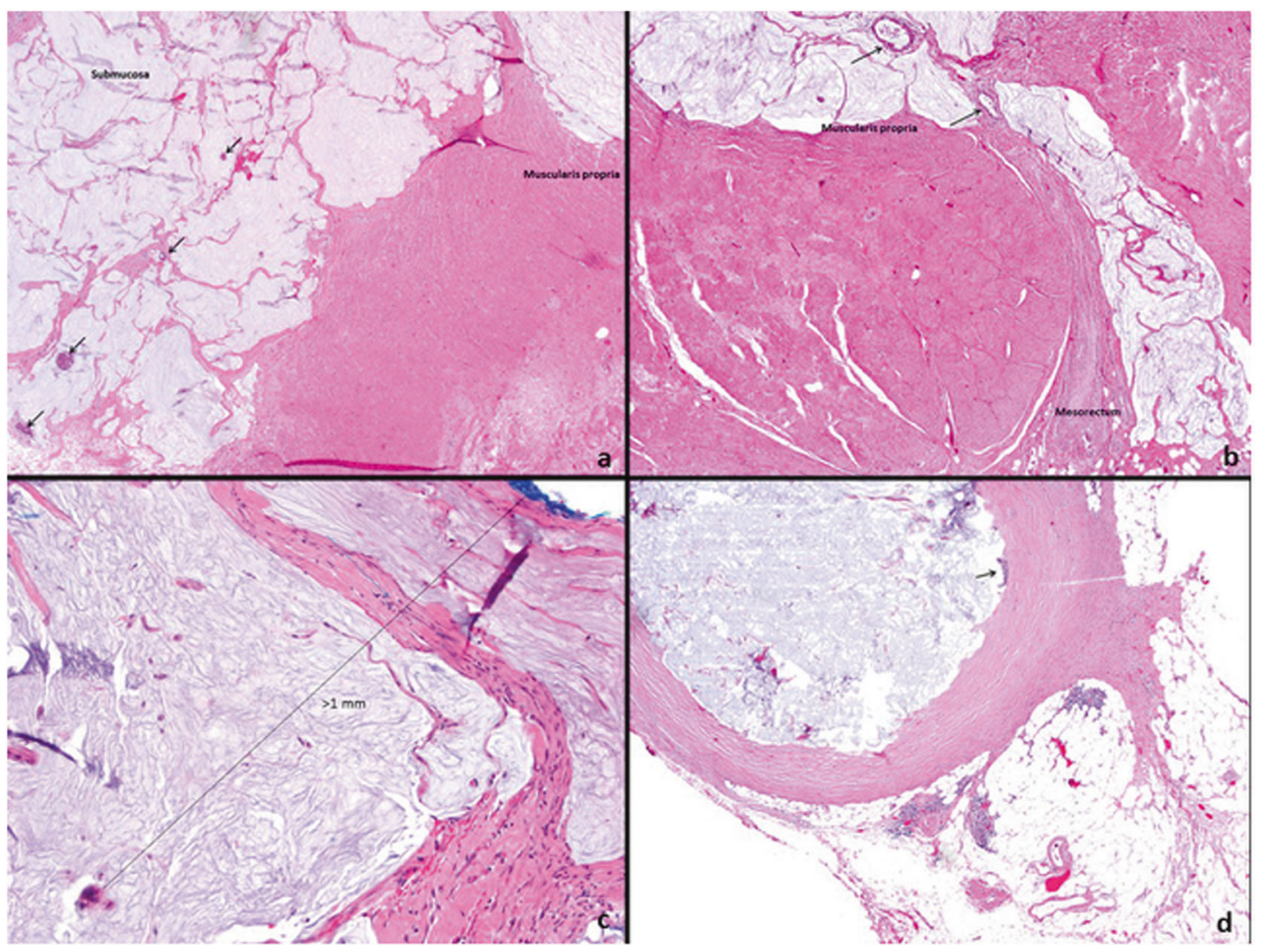

Fig. 3 Staging of neoadjuvant-treated rectal carcinoma (all these figures were included in the survey). a Viable tumor cells (arrow) floating in mucin pools in the submucosa, with the same mucin pools (with no epithelium) extending into the muscularis propria was staged as ypT1 by $65 \%$ respondents, and $35 \%$ staged ypT2. b Viable tumor cells (arrow) floating in mucin pools identified in the muscle wall, but with the same mucin pools extending into the mesorectum was staged as ypT 2 by $63 \%$ and as ypT 3 by $37 \%$. c Viable tumor cells in the mucin pools identified in the mesorectal fat ( $>1 \mathrm{~mm}$ from the radial margin), with the same mucin pool extending up to the radial margin was considered as positive radial margin by $33 \%$ of respondents. d Lymph node of treated rectal cancer show only viable tumor (measuring $<0.2 \mathrm{~mm}$, marked by arrow) within a big mucin pool. $76 \%$ staged this as ypN1 and $24 \%$ assigned ypN0 (with isolated tumor cells). (a, b, d H\&E ×100; c H\&E ×200)

invasion of tumor through areas of inflammation to the surface of the visceral peritoneum)" [2,3]. The manual also specifies that tumor present at the serosal surface, free tumor cells on the serosal surface with underlying erosion/ ulceration of mesothelial lining, mesothelial hyperplasia and/or inflammatory reaction and perforation in which the tumor cells are continuous with the serosal surface through inflammation are considered to represent pT4a disease $[2,3]$. Our findings reinforce those of others: many pathologists reject the notion that a pT4a designation requires tumor cells at the serosal surface. Several studies have shown that serosal involvement is often underestimated and that tumors $<1 \mathrm{~mm}$ from the serosa that are accompanied by a serosal reaction are at higher risk of peritoneal recurrence [19-24]. Panarelli et al. evaluated serosal cytologic smears from 120 colon cancer resection specimens and showed that pT3 tumors close $(\leq 1 \mathrm{~mm})$ to a serosal reaction were associated with positive cytology specimens from the peritoneal surface almost as frequently as pT4a tumors (55\%) [20]. Snaebjornsson et al. evaluated 


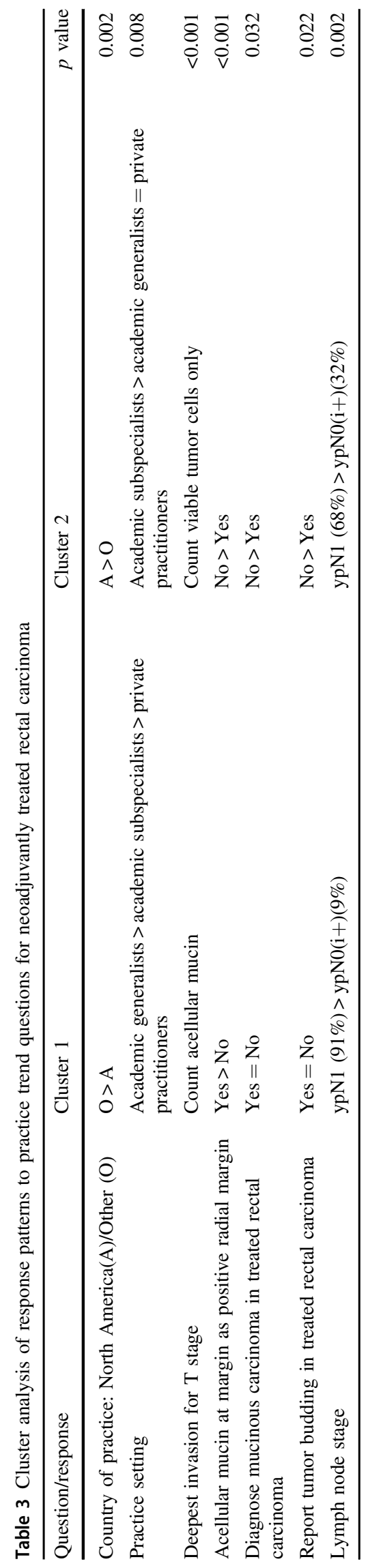

5-year survival among 889 colon cancer patients and found 5-year survival rates to be $71 \%$ for patients with pT3 tumors $>1 \mathrm{~mm}$ from the serosa, compared with $58 \%$ for patients with tumors $\leq 1 \mathrm{~mm}$ from a serosal reaction, and $51 \%$ for tumors at the serosal surface [14]. Given the available data, it seems reasonable for pathologists to convey information in pathology reports indicating that pT3 tumors within 1 $\mathrm{mm}$ of a serosal reaction are at increased risk of peritoneal spread compared with other pT3 tumors.

Categorization of isolated tumor cells and small $(<0.2 \mathrm{~mm})$ nodal tumor deposits is another area of variability. The College of American Pathologists guidelines state that "because the biologic significance of isolated tumor cells (either a single focus in a single node, multiple foci within a single, or multiple nodes) remains unproven, $\mathrm{pN} 0$ is considered justified" [3]. However, these data are largely based on data transferred to the gastrointestinal tract from the breast. While rare studies have shown that the isolated tumor cells do not affect disease recurrence [25], others have yielded different results. Protic et al. evaluated regional lymph nodes from 203 resected stage II colorectal cancers with adequate lymph node dissections (i.e., $\geq 12$ lymph nodes), which were negative by routine histologic examination and multiple tissue levels. They found that patients with isolated tumor cells in lymph nodes detected by ancillary cytokeratin immunohistochemistry had a shorter disease-free survival than patients with cytokeratinnegative lymph nodes, suggesting that presence of isolated tumor cells should not be ignored [26]. Similarly Sargent et al. evaluated histologically negative lymph nodes from 181 stage II colorectal carcinoma patients using reversetranscriptase polymerase chain reaction for guanylyl cyclase, a relatively specific protein for gastrointestinal epithelium, and found that patients with detectable guanylyl cyclase in their lymph nodes had a higher 5-year disease recurrence compared with those who had little or no detectable guanylyl cyclase in their lymph nodes [27]. Our survey data indicate that many surgical pathologists do not endorse the idea of classifying small lymph node deposits as pNO. Clearly, this is an area in great need of data-driven recommendations.

Acellular mucin deposits are occasionally detected in resected lymph nodes from cancer patients who have not received neoadjuvant therapy. Although the American Joint Committee on Cancer 8th edition Cancer Staging Manual recommends that mucin in regional lymph node be staged as $\mathrm{pN} 0$ when detected in association with appendiceal mucinous neoplasms, it does not address the finding of mucin pools in treatment-naive colorectal cancers [28]. However, the Union for International Cancer Control states that, in the absence of neoadjuvant therapy, acellular mucin pools in lymph nodes should be staged as N1 or higher depending on the number of affected lymph nodes [29-31]. 
Some experts recommend that such findings be evaluated with multiple tissue levels and submission of all pericolonic fat for histologic evaluation; and failure to identify unequivocal carcinoma cells should result in assignment of pNO [9]. Our data suggest that nearly two-thirds of respondents stage acellular mucin in lymph nodes from untreated carcinomas as $\mathrm{pN} 0$, similar to results from another survey in which more than $90 \%$ of North American pathologists considered such cases to represent pN0 disease [16]. Notably, more European pathologists stage acellular mucin deposits in lymph nodes as $\mathrm{pN} 1$ in accordance with Union for International Cancer Control guidelines. Future efforts should be aimed at coordinating the prevailing views of the American Joint Committee on Cancer and Union for International Cancer Control and basing recommendations on outcome data, if available.

Pathological assessment of neoadjuvantly treated rectal cancer resection specimens can be problematic. It is well known that circumferential resection margin status is the most important factor in predicting local recurrence and survival [32-34]. Both the American Joint Committee on Cancer 8th edition cancer-staging manual and College of American Pathologists colorectal carcinoma protocol indicate that acellular mucin should not considered when assessing depth of invasion and lymph node metastases $[2,3]$. These recommendations are based on data demonstrating a correlation between low pathologic stage based on extent of viable tumor and recurrence free survival, and no relationship between extent of acellular mucin pools and outcome [35-37]. On the other hand, Cienfuegos et al. showed that the presence of neoplastic cells in mucin pools is associated with a $17.8 \%$ and $16.9 \%$ decrease in 5- and 10year disease-free survival, respectively, when compared with cases with acellular mucin pools alone [38]. Although most pathologists in the United States assign stage and margin status based on the extent of viable tumor cells rather than mucin as recommended by the American Joint Committee on Cancer 8th edition cancer-staging manual, $\sim 35 \%$ still assign stage based on extent of mucin pools.

The results of our study illustrate a considerable lack of uniformity among experienced pathologists with respect to execution of staging criteria set forth by the American Joint Committee on Cancer 8th edition cancer-staging manual and draw attention to areas that require further study. A weakness of this study is the inability to determine whether variations in reporting are due to a lack of awareness of new/changed criteria in the American Joint Committee on Cancer 8th edition staging manual, or differing application of staging criteria because of differing endorsements among local/national-governing bodies or individual practice variations. There may also be uncertainty over which guideline to use. Notably, a recent survey involving predominantly European pathologists showed that there is a wide variability among pathologists regarding which guideline they use and how strictly they follow individual recommendations in their daily practice [39]. So, in addition to updating the guidelines, reinforcing the adherence to guidelines is also needed to reduce variability and ensure the best standards for reporting colorectal cancer specimens [39]. In addition, the survey did not ask respondents to clarify which staging system they follow, and whether they are familiar with the American Joint Committee on Cancer 8 th edition staging manual. The American Joint Committee on Cancer 8th edition staging manual is relatively new (January 01, 2018), and it is possible that some pathologists were not aware of the updated guidelines.

One of the strengths of this study is that this was an international study and used questions and case scenarios with selected figures targeting the latest American Joint Committee on Cancer 8th edition staging manual protocols. Some of the findings in this study paralleled those what were found in an older survey and serves to reiterate that these are recurring issues with interpretation [16]. In addition, to ensure that the data presented in this study were valid, we tried to assess whether this number of 118 respondents was sufficiently powered for the aims of the study. The purpose of this study was largely descriptive: to assess how pathologists apply cancer-staging criteria when faced with problematic issues that are not well delineated or supported by data. We also assessed whether reporting practices were influenced by regional effects, different practice settings, or years of experience, and due to the nature of this international survey, all available respondents were included in the statistical analysis. Most statisticians feel that post hoc power calculations are not very meaningful as they are just functions of the observed $p$-value. However, in general a sample size of 118 will provide a level of precision around an estimate of a proportion of $\sim \pm 0.08$, which supports the assumption that meaningful estimates can result from a study of this size [40].

To conclude, this survey highlighted several "gray zones" subject to interpretive bias. Some reporting issues are not addressed by the American Joint Committee on Cancer 8th edition staging manual/College of American Pathologists colorectal carcinoma protocol, such as staging of nodal acellular mucin in treatment-naive colorectal carcinoma. There remain difficulties with certain scenarios, such as choosing between T3 and T4a in certain cases. Finally, it appears that in some cases pathologists choose to disregard guidelines, such as staging of small nodal tumor deposits $(<0.2 \mathrm{~mm})$ as N0, probably because they believe that the recommendation is not data-based. Data-driven recommendations, particularly in regards to staging issues around isolated tumor cells and outcome studies of untreated patients with acellular mucin in lymph nodes, as well as a greater degree of clarity regarding staging criteria 
are needed to reduce subjectivity and ensure consistent, reproducible, and accurate-staging information for patients with colorectal carcinoma.

\section{Compliance with ethical standards}

Conflict of interest The authors declare that they have no conflict of interest.

Publisher's note: Springer Nature remains neutral with regard to jurisdictional claims in published maps and institutional affiliations.

\section{References}

1. Siegel RL, Miller KD, Jemal A. Cancer statistics, 2019. CA Cancer J Clin. 2019;69:7-34.

2. Jessup JM, Goldberg RM, Asare EA, Benson III AB, Brierley JD, Chang GJ. et al. Colon and rectum. In: Amin MB (ed.) AJCC cancer staging manual. 8th ed Chicag: Springer; 2017. p. 251-74.

3. Kakar S, Shi C, Berho ME, Driman DK, Fitzgibbons P, Frankel WL, et al. College of American Pathologists Protocol for the Examination of Specimens from patients with primary carcinoma of the colon and rectum. (Version: Colon Rectum 4.0.1.0, Protocol Posting Date: June 2017). https://documents.cap.org/ protocols/cp-gilower-colonrectum-17protocol-4010.pdf.

4. Dawson H, Kirsch R, Messenger D, Driman D. A review of current challenges in colorectal cancer reporting. Arch Pathol Lab Med. 2019. https://doi.org/10.5858/arpa.2017-0475-RA.

5. Frankel WL, Jin M. Serosal surfaces, mucin pools, and deposits, oh my: challenges in staging colorectal carcinoma. Mod Pathol. 2015;28:S95-108.

6. Maguire A, Sheahan K. Controversies in the pathological assessment of colorectal cancer. World J Gastroenterol. 2014; 20:9850-61.

7. Puppa G, Sonzogni A, Colombari R, Pelosi G. TNM staging system of colorectal carcinoma: a critical appraisal of challenging issues. Arch Pathol Lab Med. 2010;134:837-52.

8. Shia J, Klimstra DS, Bagci P, Basturk O, Adsay NV. TNM staging of colorectal carcinoma: issues and caveats. Semin Diagn Pathol. 2012;29:142-53.

9. Yantiss RK. Persistent problems in colorectal cancer reporting. Surg Pathol Clin. 2017;10:961-76.

10. Benson AB 3rd, Schrag D, Somerfield MR, Cohen AM, Figueredo AT, Flynn PJ, et al. American Society of Clinical Oncology recommendations on adjuvant chemotherapy for stage II colon cancer. J Clin Oncol. 2004;22:3408-19.

11. Williams CD, Grady WM, Zullig LL. Use of NCCN guidelines, other guidelines, and biomarkers for colorectal cancer screening. $\mathrm{J}$ Natl Compr Cancer Netw. 2016;14:1479-85.

12. Dotan E, Cohen SJ. Challenges in the management of stage II colon cancer. Semin Oncol. 2011;38:511-20.

13. Benson AB, Venook AP, Al-Hawary MM, Cederquist L, Chen YJ, Ciombor KK, et al. NCCN guidelines insights: colon cancer, version 2.2018. J Natl Compr Canc Netw. 2018;16:359-69.

14. Snaebjornsson P, Coupe VM, Jonasson L, Meijer GA, van Grieken NC, Jonasson JG. pT4 stage II and III colon cancers carry the worst prognosis in a nationwide survival analysis. Shepherd's local peritoneal involvement revisited. Int J Cancer. 2014; 135:467-78.

15. Jin M, Frankel WL. Lymph node metastasis in colorectal cancer. Surg Oncol Clin N Am. 2018;27:401-12.
16. Kirsch RMD, Shepherd NA, Dawson H, Driman DK. Wide variability in assessment and reporting of colorectal cancer specimens among North American pathologists: results of a CanadaUS survey. Can J Pathol. 2019;11:59-69.

17. Compton CC. Key issues in reporting common cancer specimens: problems in pathologic staging of colon cancer. Arch Pathol Lab Med. 2006;130:318-24.

18. Athanasakis E, Xenaki S, Venianaki M, Chalkiadakis G, Chrysos E. Newly recognized extratumoral features of colorectal cancer challenge the current tumor-node-metastasis staging system. Ann Gastroenterol. 2018;31:525-34.

19. Gunderson LL, Jessup JM, Sargent DJ, Greene FL, Stewart AK. Revised TN categorization for colon cancer based on national survival outcomes data. J Clin Oncol. 2010;28:264-71.

20. Panarelli NC, Schreiner AM, Brandt SM, Shepherd NA, Yantiss RK. Histologic features and cytologic techniques that aid pathologic stage assessment of colonic adenocarcinoma. Am J Surg Pathol. 2013;37:1252-8.

21. Stewart CJ, Hillery S, Platell C, Puppa G. Assessment of serosal invasion and criteria for the classification of pathological (p) T4 staging in colorectal carcinoma: confusions, controversies and criticisms. Cancers. 2011;3:164-81.

22. Woods YL, Mukhtar S, McClements P, Lang J, Steele RJ, Carey FA. A survey of reporting of colorectal cancer in Scotland: compliance with guidelines and effect of proforma reporting. J Clin Pathol. 2014;67:499-505.

23. Swamy R. Histopathological reporting of pT4 tumour stage in colorectal carcinomas: dotting the 'i's and crossing the 't's. J Clin Pathol. 2010;63:110-5.

24. Shepherd NA, Baxter KJ, Love SB. The prognostic importance of peritoneal involvement in colonic cancer: a prospective evaluation. Gastroenterology. 1997;112:1096-102.

25. Sloothaak DA, Sahami S, van der Zaag-Loonen HJ, van der Zaag ES, Tanis PJ, Bemelman WA, et al. The prognostic value of micrometastases and isolated tumour cells in histologically negative lymph nodes of patients with colorectal cancer: a systematic review and meta-analysis. Eur J Surg Oncol. 2014;40:263-9.

26. Protic M, Stojadinovic A, Nissan A, Wainberg Z, Steele SR, Chen DC, et al. Prognostic effect of ultra-staging node-negative colon cancer without adjuvant chemotherapy: a prospective national Cancer Institute-sponsored clinical trial. J Am Coll Surg. 2015;221:643-51.

27. Sargent DJ, Resnick MB, Meyers MO, Goldar-Najafi A, Clancy $\mathrm{T}$, Gill S, et al. Evaluation of guanylyl cyclase C lymph node status for colon cancer staging and prognosis. Ann Surg Oncol. 2011;18:3261-70.

28. Overman MJ, Asare EA, Compton CC, Hanna NN, Kakar S, Kosinski LA. et al.Appendix-carcinoma. In: Amin MB (Ed.) AJCC cancer staging manual. 8th ed Chicago: Springer; 2017. p. 237-50.

29. Union for International Cancer Control. TNM frequently asked questions (FAQ'S). http://www.uicc.org/sites/main/files/atoms/ files/E_TNM_FAQs_12_16.pdf.

30. Foong KS, McGrath S, Wang LM, Shepherd NA. Reply: how do we stage acellular mucin in lymph nodes of colorectal cancer specimens without neoadjuvant therapy? Histopathology. 2017;70:507.

31. Foong KS, Mishra A, Guy R, Wang LM, Shepherd NA. How do we stage acellular mucin in lymph nodes of colorectal cancer specimens without neo-adjuvant therapy? Histopathology. 2016;69:527-8.

32. Adam IJ, Mohamdee MO, Martin IG, Scott N, Finan PJ, Johnston $\mathrm{D}$, et al. Role of circumferential margin involvement in the local recurrence of rectal cancer. Lancet. 1994;344:707-11. 
33. Chan KW, Boey J, Wong SK. A method of reporting radial invasion and surgical clearance of rectal carcinoma. Histopathology. 1985;9:1319-27.

34. Quirke P, Durdey P, Dixon MF, Williams NS. Local recurrence of rectal adenocarcinoma due to inadequate surgical resection. Histopathological study of lateral tumour spread and surgical excision. Lancet. 1986;2:996-9.

35. Shia J, McManus M, Guillem JG, Leibold T, Zhou Q, Tang LH, et al. Significance of acellular mucin pools in rectal carcinoma after neoadjuvant chemoradiotherapy. Am J Surg Pathol. 2011;35:127-34.

36. de Campos-Lobato LF, Dietz DW, Stocchi L, Vogel JD, Lavery IC, Goldblum JR, et al. Clinical implications of acellular mucin pools in resected rectal cancer with pathological complete response to neoadjuvant chemoradiation. Colorectal Dis. 2012;14:62-7.
37. Bhatti AB, Akbar A, Khattak S, Kazmi AS, Jamshed A, Syed AA. Impact of acellular mucin pools on survival in patients with complete pathological response to neoadjuvant treatment in rectal cancer. Int J Surg. 2014;12:1123-6.

38. Cienfuegos JA, Baixauli J, Rotellar F, Arredondo J, Sola JJ, Arbea $\mathrm{L}$, et al. Clinical significance of cellular and acellular mucin pools in rectal carcinoma following preoperative chemoradiotherapy. Clin Transl Oncol. 2016;18:714-21.

39. Urbanowicz M, Grabsch HI, Fiteni F, Liu Y, Caballero C, Fléjou JF. An international survey-based study on colorectal cancer pathology reporting-guidelines versus local practice. Virchows Arch. 2018;473:697-708.

40. Dixon WJ, Massey FJ. Introduction to statistical analysis. 4th ed. New York: McGraw-Hill; 1983. p. 105-7. 